\title{
Analytical Study of QAM With Interference Cancellation for High-Speed Multicode CDMA
}

\author{
Bin Xia and Jiangzhou Wang, Senior Member, IEEE
}

\begin{abstract}
In this paper, we consider the multilevel quadrature amplitude modulation (M-QAM) for forward link of multicode code-division multiple-access (CDMA) systems with interference cancellation to support high data rate service, and provide an analytical bit error rate (BER) performance of the system. In the current third-generation (3G) wide-band CDMA systems, in addition to multicode transmission, M-QAM is employed for high-speed downlink packet access (HSPDA) due to its high spectral efficiency. In frequency-selective fading channels, multipath interference seriously degrades the system performance, therefore interference cancellation technique is employed at the receiver. In this paper, an analytical closed-form BER of multicode CDMA systems with QAM is presented, where two important factors (channel estimation error and additive interference) on system performance are considered. Numerical results show that the system is very sensitive to additive multipath interference. With the help of interference cancellation technique, M-QAM can be adaptively employed in high signal-to-noise ratio cases to increase system throughput, within 5 MHz bandwidth. Meanwhile, simulation results are shown to illustrate the accuracy of the analytical method. Moreover, it is found that extra pilot power should be invested for more accurate channel estimation and consequently better BER performance when using the interference cancellation technique.
\end{abstract}

Index Terms-Channel estimation, interference cancellation, multicode code-division multiple access (CDMA), multipath interference, quadrature amplitude modulation (QAM).

\section{INTRODUCTION}

W IDE-BAND code-division multiple-access (WCDMA) technique has gained much attention in the past several years. To support different high-quality data services with various bit rates, multicode transmission has been considered to satisfy the requirement and has been adopted in 3GPP [1]. On the demand of much higher speed package transmission in forward link, in addition to multicode transmission, multilevel quadrature amplitude modulation (M-QAM) has also been employed for high-speed downlink packet access (HSPDA) due to its high spectral efficiency.

In the present WCDMA system, channalization codes are generated from orthogonal variable spreading factor (OVSF) codes [2] whose orthogonal properties make different channels not cause interference to each other in a Gaussian or flat fading channel. However, the performance of a multicode WCDMA

Manuscript received March 27, 2004; revised October 31, 2004. The review of this paper was coordinated by Dr. Y.-P. E. Wang.

The authors are with the Department of Electrical and Electronic Engineering, University of Hong Kong, Hong Kong, China (e-mail: bxia@eee.hku.hk; jwang@eee.hku.hk).

Digital Object Identifier 10.1109/TVT.2005.844656 system under frequency-selective fading channels seriously degrades due to nonorthogonality of different code channels in the presence of different multipath delays. Several studies have been done to combat the multipath interference using interference regeneration and subtraction method, which is known as interference cancellation. The multistage interference cancellation scheme was first proposed in asynchronous CDMA system by Varanasi and Aazhang [3]. It has been extensively studied because of its low complexity and performance improvement [4]-[8].

The concept of high-order modulation for CDMA systems was first proposed by Milstein et al. [9], [10] to support higher data rate and multimedia transmission for wireless communications. Performance on a coherent Rake receiver for 16-QAM modulated single code CDMA in frequency-selective fading channel was evaluated by means of simulation in [11]. It has been shown in [12]-[14] that M-QAM is very sensitive to the amplitude and phase offset fluctuations. Recent studies on the M-QAM modulated CDMA systems assumed perfect channel estimation [11], [15]-[17]. Some others are based on computer simulations [12], [13]. The only analytical result of QAM in a flat fading channel was presented by Tang et al. [14], but multipath effect and multicode CDMA transmission were not involved.

In [18], the performance of the QAM modulated multicode WCDMA system in the presence of multipath interference with Rake receiver and interference cancellation has been investigated. But no explicit BER analytical results have been given. In this paper, we present an analytical study of the QAM multicode WCDMA system with multistage interference cancellation. Using the well-known characteristic function method, a closed form bit error rate (BER) solution is derived.

This paper is organized as follows. In Section II, the system model is presented, including transmitter, channel, and multistage parallel interference cancellation coherent Rake receiver models. In Section III, a pilot aided channel estimator is described. The BER performance analysis is presented in Section IV. In Section V, comparison and discussion on numerical BER results with variable parameters are presented. Finally, some conclusions are drawn in Section VI.

\section{SYSTEM MODELS}

\section{A. Transmitter Model}

Similar to [1], the transmitter diagram of the forward link for a QAM multicode WCDMA system is shown in Fig. 1. A common pilot channel is employed separately from data channels. The source data are first divided into $K$ streams and 


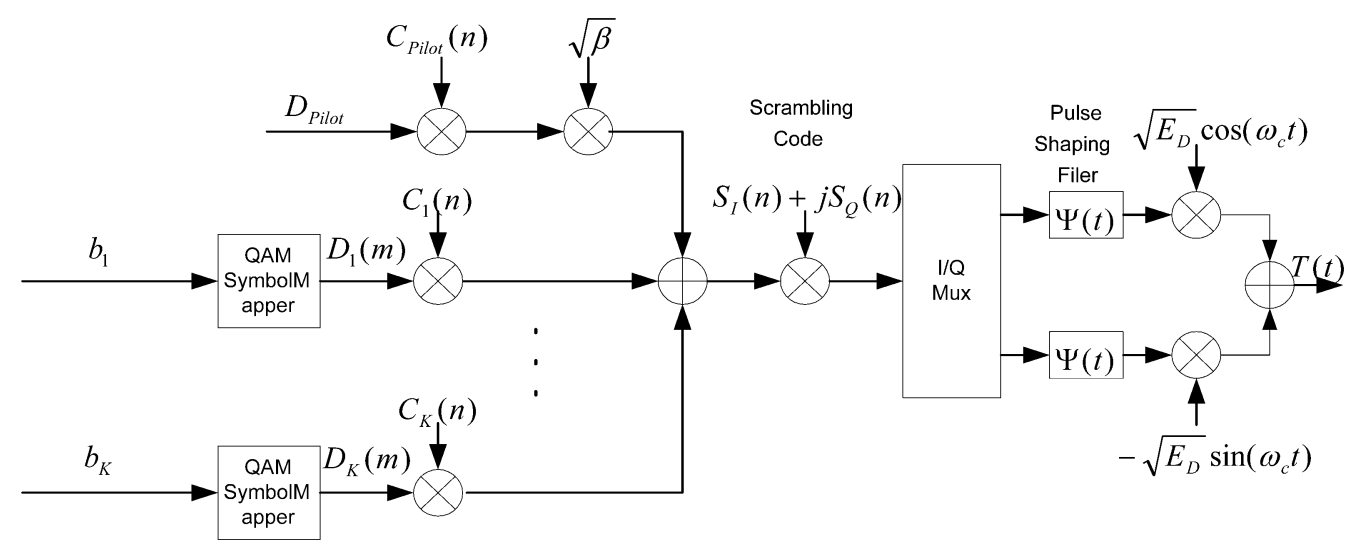

Fig. 1. Transmitter diagram of QAM modulated multicode CDMA systems.

TABLE I

THE INPUT/OUTPUT RELATIONSHIP OF GRAY CODER FOR 16-QAM

\begin{tabular}{c|c|c|c|c}
\hline Input Bits (Code word) & 01 & 00 & 10 & 11 \\
\hline $\begin{array}{c}\text { Output Coded Symbol } \\
D_{k}(m)\end{array}$ & $\frac{-3}{\sqrt{5 / 2}}$ & $\frac{-1}{\sqrt{5 / 2}}$ & $\frac{1}{\sqrt{5 / 2}}$ & $\frac{3}{\sqrt{5 / 2}}$ \\
\hline
\end{tabular}

fed into $K$ M-QAM symbol mappers before spreading. The complex data symbols $\left\{D_{k}(m)\right)$ are multiplied with real valued OVSF codes $\left\{C_{k}(n)\right\}$ for the channelization operation. The resultant signals, including pilot, are multiplied by a complex valued code for scrambling. Then, the scrambled signal is shaped by a chip waveform shaping filter and up-converted for suitable transmission over wireless channels.

At the M-QAM mapper, as shown in [14], the binary data stream is first split into in-phase (I) and quadrature (Q) branches for independent Gray encoding. Then the coded symbol streams are combined into a complex symbol stream. Table I shows the Gray codes and amplitudes of a 2-bit code word for 16-QAM.

The transmitted signal can be written as

$$
\begin{aligned}
T(t)=\operatorname{Re}\left\{\sqrt{E_{D}} e^{-j \omega_{c} t}\right. \\
\times \sum_{n=-\infty}^{\infty}\left(\sqrt{\beta} D_{\text {Pilot }} C_{\text {Pilot }}(n)\right. \\
\left.\left.\quad+\sum_{k=1}^{K} D_{k}(m) C_{k}(n)\right) S(n) \Psi\left(t-n T_{c}\right)\right\}
\end{aligned}
$$

where

$E_{D} \quad$ average chip energy of each data channel.

$\beta \quad$ power ratio of pilot channel to one data channel.

$C_{k}(n) \quad$ real-valued OVSF channelization code with amplitude taking real values \pm 1 and chip duration $T_{c}$. Therefore, spreading factor for data channel $N=T_{D} / T_{c}$.

$C_{\text {Pilot }}(n)$ real-valued orthogonal user-specific channelization code for pilot channel. In the WCDMA system it is always unitary $\left(C_{\text {Pilot }}(n)=1\right)$.

$S(n) \quad$ complex-valued cell-specific code (scrambling code) having complex value $S(n)=$ $S_{I}+j S_{Q}= \pm 1 \pm j, j=\sqrt{-1}$.
$D_{k}(m) \quad m$ th complex valued data symbol with symbol duration $T_{D}$ and $D_{k}(m)= \pm\left(2 k_{1}-\right.$ 1) $\pm j\left(2 k_{2}-1\right) / \sqrt{2(M-1) /\left(3 \log _{2} M\right)}$, $k_{1}, k_{2} \in\{1,2, \ldots, \sqrt{M} / 2\}$.

$D_{\text {Pilot }} \quad$ known symbol $D_{\text {Pilot }} \equiv 1+j$ in pilot channel for channel estimation and preprocessing in the receiver.

$m \quad$ data symbol order in time domain, $m=\lfloor n / N\rfloor$, i.e., $m$ takes integer of $n / N$.

$\Psi(t) \quad$ real-valued impulse response of the band-limited chip shaping filter with $\int_{-\infty}^{\infty} \Psi^{2}(t) d t=1$.

\section{B. Channel Model}

The channel is assumed to be a multipath fading channel. When the fading rate is much slower than the symbol rate, the channel remains constant over a few symbols' duration. This is particularly true since a mobile terminal does not move fast for high data rate transmission. The complex low-pass equivalent impulse response of such a multipath fading channel can be written as

$$
h(t)=\sum_{l=1}^{L} \alpha_{l}(t) \delta\left(t-\tau_{l}(t)\right) e^{j \theta_{l}(t)}
$$

where $L$ is the number of resolvable paths, $\tau_{l}(t)$ is the delay of the $l$ th path, $\theta_{l}(t)$ is the phase, uniformly distributed in $[0,2 \pi)$, and $\alpha_{l}(t)$ is the fading amplitude, Rayleigh distributed with probability density function (pdf)

$$
p\left(\alpha_{l}(t)\right)=\frac{2 \alpha_{l}(t)}{\Omega_{l}} e^{-\alpha_{l}^{2}(t) / \Omega_{l}} \quad \alpha_{l}>0
$$

where $\Omega_{l}$ is the average fading power of each path, i.e.,

$$
\Omega_{l}=E\left\{\alpha_{l}^{2}(t)\right\} .
$$




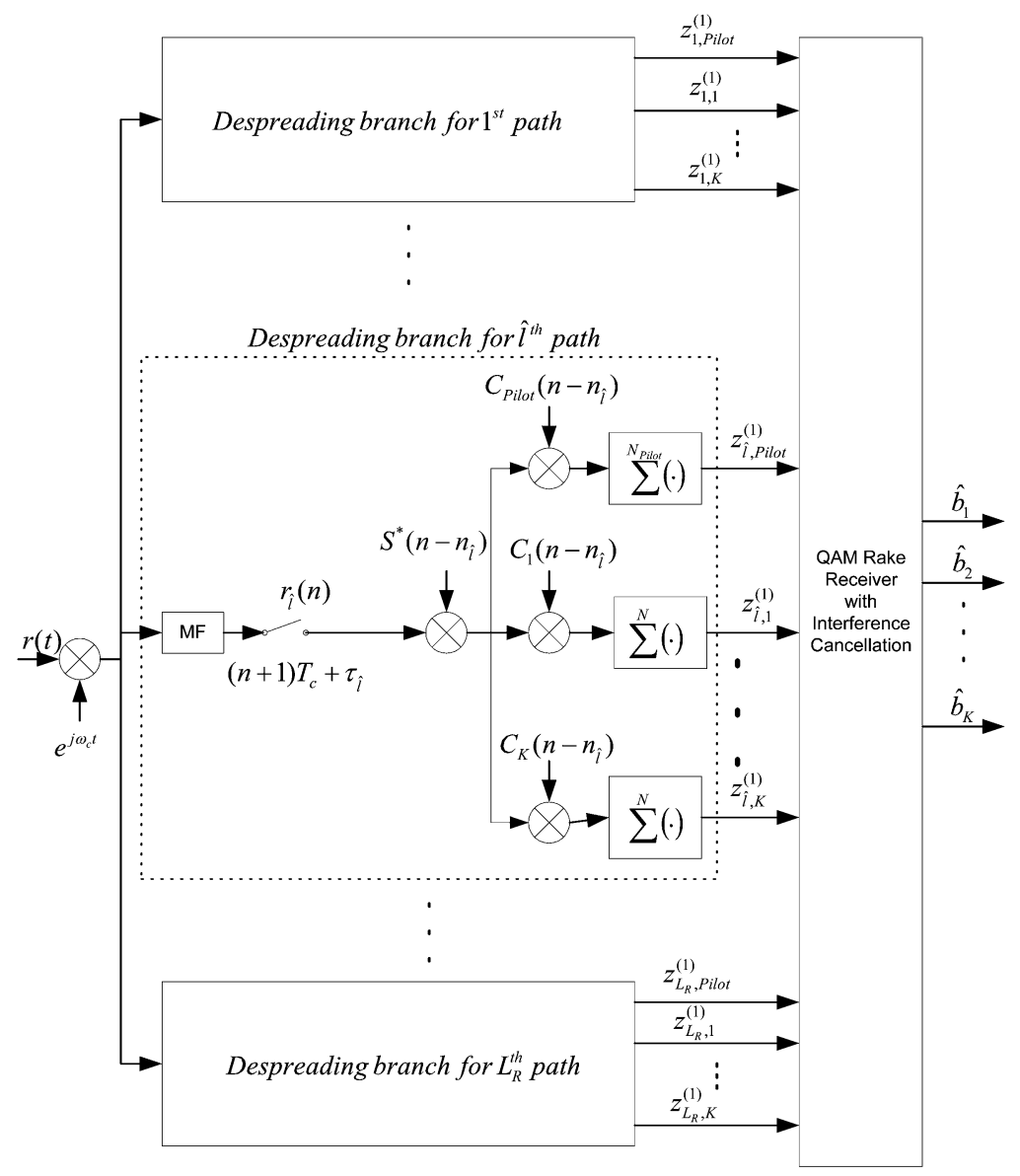

Fig. 2. Receiver diagram of QAM modulated multicode CDMA systems.

All random variables in (2) are assumed to be independent. Since $\alpha_{l}(t), \theta_{l}(t)$, and $\tau_{l}(t)$ are constant over a few symbols, " $t$ " is simply omitted for simplicity of notation, unless otherwise noted.

\section{Receiver Model}

The received signal can be written as

$$
r(t)=\sum_{l=1}^{L} \alpha_{l} \hat{T}\left(t-\tau_{l}\right)+\eta(t)
$$

where $\eta(t)$ is the background bandpass additive white Gaussian noise (AWGN) with double-sided spectral density $\left(N_{0} / 2\right)$. $\hat{T}\left(t-\tau_{l}\right)$ is given by (1) with the substitution of $\omega_{c} t$ by $\omega_{c} t-\theta_{l}$.

Although the transmitted signal experiences an $L$-path fading channel, only the $L_{R}\left(L_{R} \leq L\right)$ strongest paths are used at the Rake receiver. As shown in Fig. 2, the down-converted signal is fed into $L_{R}$ despreading branches corresponding to the $L_{R}$ strongest paths. In each branch, the sampled signal after chipmatched filter is descrambled and then passes through $K+1$ correlators ( $K$ data and one pilot) for despreading. Finally, the outputs are fed into the QAM Rake receiver with interference cancellation block, which makes use of the regeneration properties of multipath interference and mitigates the multipath effect. The detailed diagram of the QAM Rake receiver is shown in
Fig. 3, where the estimated channel fading and phase of the $s$ th stage $\hat{z}_{\hat{l}, \text { Pilot }}^{(s)}$ for the $\hat{l}$ th path are used to eliminate the phase error and achieve maximum ratio combining (MRC), meanwhile, to scale the combining output for M-QAM symbol demodulation. $\left\{z_{\hat{\imath}, \hat{k}}^{(1)}\right\}$ are the original inputs to all stages for the $\hat{k}$ th desired code, whereas $\left\{z_{\hat{l}, \hat{k}}^{(s)}\right\}$ are the inputs at the sth stage after $\left\{z_{\hat{l}, \hat{k}}^{(1)}\right\}$ are filtered by an interference cancellation block.

From Fig. 2, the signals of the $\hat{l}$ th path after the matched filter can be written as

$$
r_{\hat{l}}(n)=\int_{n T_{c}+\tau_{\hat{l}}}^{(n+1) T_{c}+\tau_{\hat{\imath}}} r(t) e^{j \omega_{c} t} \Psi^{*}\left(t-n T_{c}-\tau_{\hat{l}}\right) d t .
$$

Neglecting the high-frequency components, the sampled signal can be further written as

$$
\begin{aligned}
r_{\hat{l}}(n)= & \frac{1}{2} \sum_{l=1}^{L} \alpha_{l} e^{j \xi_{l}} \sum_{i=-\infty}^{\infty} R\left(\bar{\tau}_{l, \hat{l}}-i T_{c}\right) \\
& \times\left(\sqrt{\beta E_{D}} D_{\text {Pilot }} C_{\text {Pilot }}\left(n-n_{l, i}\right)\right. \\
& \left.\quad+\sum_{k=1}^{K} \sqrt{E_{D}} D_{k}\left(m_{l, i}\right) C_{k}\left(n-n_{l, i}\right)\right) \\
& \times S\left(n-n_{l, i}\right)+\eta_{\hat{l}}(n)
\end{aligned}
$$




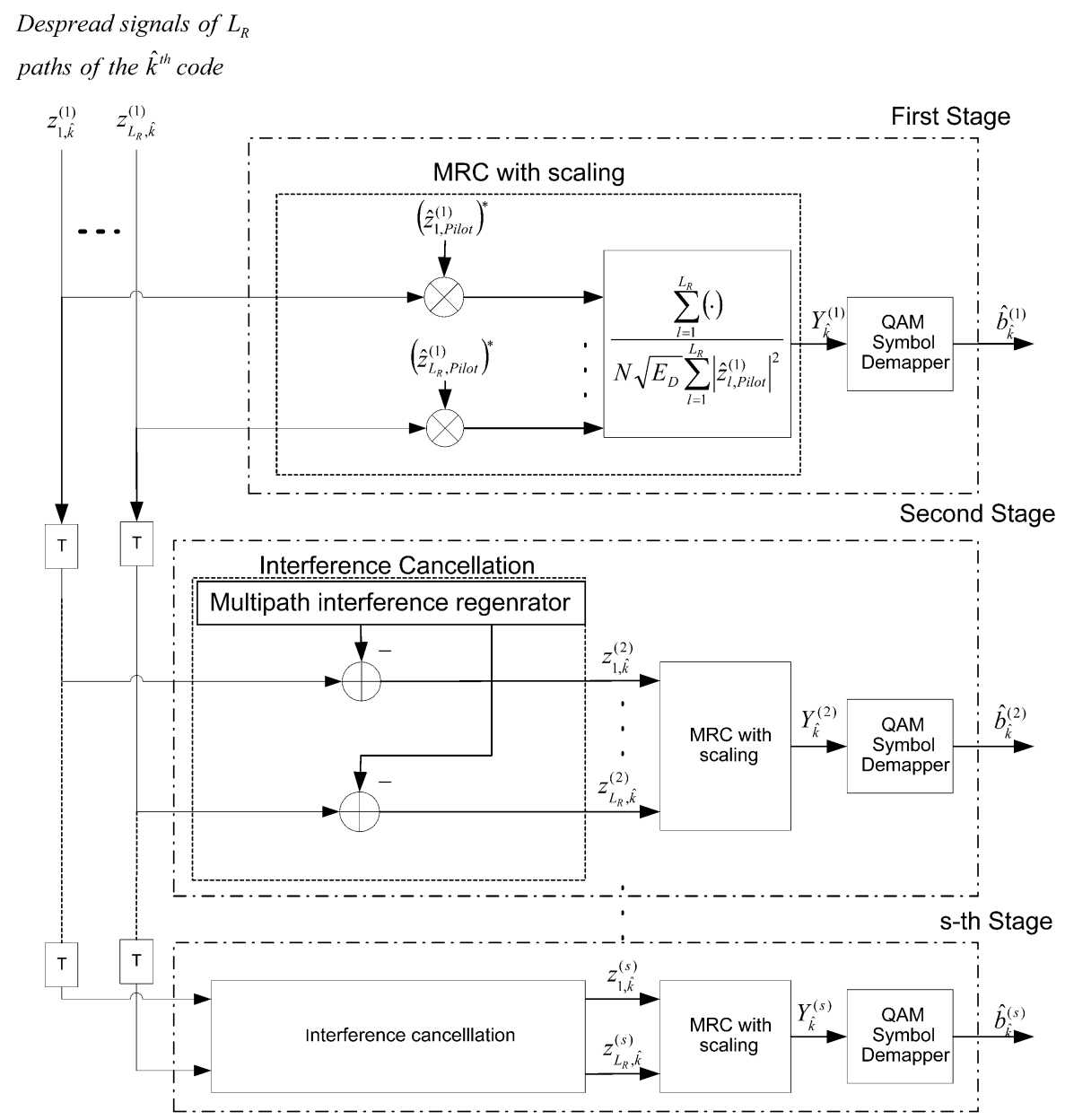

Fig. 3. Detailed block diagram of QAM Rake receiver with interference cancellation for the $\hat{k}$ th code channel.

where $\xi_{l}=\theta_{l}-\omega_{c} \tau_{l}$ is the independent identically distributed resident phase error and uniformly distributed in $[0,2 \pi) . \eta_{\hat{l}}$ is the complex Gaussian random noise with covariance function

$$
E\left\{\eta_{\hat{l}}(t) \eta_{\bar{l}}^{*}(\tau)\right\}=N_{0} \delta(t-\tau)
$$

where $\delta(t-\tau)=1$ and 0 for $t=\tau$ and $t \neq \tau$, respectively. In (7), $n_{l, i}=\left\lfloor\tau_{l} / T_{c}\right\rfloor-i$, and $m_{l, i}=\left\lfloor n-n_{l, i} / N\right\rfloor$, $\bar{\tau}_{l, \hat{l}}=\tau_{l}-\tau_{\hat{l}}-\left\lfloor\tau_{l}-\tau_{\hat{l}} / T_{c}\right\rfloor T_{c}$ is the chip timing error, $R\left(\bar{\tau}_{l, \hat{l}}\right)$ is the effect of timing error $\bar{\tau}_{l, \hat{l}}$ on the output, and $R\left(\bar{\tau}_{l, \hat{l}}\right)=$ $\int_{-\infty}^{\infty}|\Psi(f)|^{2} \cos \left(2 \pi f \bar{\tau}_{l, \hat{l}}\right) d f[20]$. Note that $R\left(i T_{c}\right)=1$ and 0 for $i=0$ and other nonzero integers, respectively. Actually, $R(t)$ is very small as long as $t$ is greater than a few chip intervals.

Then, the output of the $\hat{l}$ th despreading branch for the $\hat{k}$ th desired code $z_{\hat{l}, \hat{k}}^{(1)}$ can be written by

$$
\begin{aligned}
z_{\hat{l}, \hat{k}}^{(1)}= & \frac{1}{2} \sqrt{E_{D}} \\
& \times \sum_{k=1}^{K} \sum_{l=1}^{L} \alpha_{l} e^{j \xi_{l}} \sum_{i=-\infty}^{\infty} \sum_{n=m N+n_{\hat{\imath}}}^{(m+1) N+n_{\hat{\imath}}-1} R\left(\bar{\tau}_{l, \hat{l}}-i T_{c}\right) \\
& \times D_{k}\left(m_{l, i}\right) C_{k}\left(n-n_{l, i}\right) S\left(n-n_{l, i}\right) \\
& \times C_{\hat{k}}\left(n-n_{\hat{l}}\right) S^{*}\left(n-n_{\hat{l}}\right) \\
& +\frac{1}{2} \sqrt{\beta E_{D}}
\end{aligned}
$$

$$
\begin{aligned}
& \times \sum_{l=1}^{L} \alpha_{l} e^{j \xi_{l}} \sum_{i=-\infty}^{\infty} \sum_{n=m N+n_{\hat{\imath}}}^{(m+1) N+n_{\hat{\imath}}-1} R\left(\bar{\tau}_{l, \hat{l}}-i T_{c}\right) \\
& \times D_{\text {Pilot }} C_{\text {Pilot }}\left(n-n_{l, i}\right) S\left(n-n_{l, i}\right) \\
& \times C_{\hat{k}}\left(n-n_{\hat{l}}\right) S^{*}\left(n-n_{\hat{l}}\right) \\
& +\sum_{n=m N+n_{\hat{\imath}}}^{(m+1) N+n_{\hat{\imath}}-1} \eta_{\hat{l}}(n) C_{\hat{k}}\left(n-n_{\hat{l}}\right) S^{*}\left(n-n_{\hat{l}}\right) \\
= & U_{s, \hat{l}, \hat{k}}(m)+U_{p, \hat{l}, \hat{k}}^{(1)}(m)+U_{\mathrm{Pilot} \hat{l}, \hat{k}}^{(1)}(m) \\
& +U_{\mathrm{AWGN}, \hat{l}, \hat{k}}(m)
\end{aligned}
$$

where $U_{s, \hat{l}, \hat{k}}(m)$ is the desired signal component, which is from the $\hat{l}$ th path of the $\hat{k}$ th code

$$
U_{s, \hat{l}, \hat{k}}(m)=N \alpha_{\hat{\imath}} \sqrt{E_{D}} D_{\hat{k}}(m) e^{j \xi_{\hat{\imath}}} .
$$

The desired signal component is related to the channel fading gain and phase. Thus, the inputs of the M-QAM demapper should be scaled by the estimated $N \alpha_{\hat{l}} \sqrt{E_{D}} e^{j \xi_{\hat{\imath}}}$ for symbol decision according to the constellation map. If the channel fading gain or phase is estimated incorrectly, the improper scaling will lead to incorrect M-QAM demodulation [14]. Therefore, QAM-based WCDMA systems require for good fading scaling compensation at the receiver. 
$U_{p, \hat{l}, \hat{k}}^{(1)}(m)$ is the multipath interference term. Since the multipath interference from the same path ( $\hat{l}_{\text {th }}$ path) is zero due to the orthogonality of the channel codes, the total multipath interference can be written as

$$
\begin{aligned}
U_{p, \hat{l}, \hat{k}}^{(1)}(m)= & \frac{1}{2} \sqrt{E_{D}} \sum_{k=1}^{K} \sum_{\substack{l=1 \\
l \neq \hat{l}}}^{L} \alpha_{l} e^{j \xi_{l}} \\
& \times \sum_{i=-\infty}^{\infty} \sum_{n=m N+n_{\hat{\imath}}}^{(m+1) N+n_{\hat{\imath}}-1} R\left(\bar{\tau}_{l, \hat{l}}-i T_{c}\right) \\
& \times D_{k}\left(m_{l, i}\right) C_{k}\left(n-n_{l, i}\right) S\left(n-n_{l, i}\right) \\
& \times C_{\hat{k}}\left(n-n_{\hat{l}}\right) S^{*}\left(n-n_{\hat{\imath}}\right) .
\end{aligned}
$$

$U_{\text {Pilot, }, \hat{l}, \hat{k}}^{(1)}(m)$ is the interference from pilot channel, given by

$$
\begin{aligned}
U_{\text {Pilot }, \hat{l}, \hat{k}}^{(1)}(m)= & \frac{1}{2} \sqrt{\beta E_{D}} \sum_{\substack{l=1 \\
l \neq \hat{l}}}^{L} \alpha_{l} e^{j \xi_{l}} \\
& \times \sum_{i=-\infty}^{\infty} \sum_{n=m N+n_{\hat{\imath}}}^{(m+1) N+n_{\hat{\imath}}-1} R\left(\bar{\tau}_{l, \hat{l}}-i T_{c}\right) \\
& \times D_{\text {Pilot }} C_{\text {Pilot }}\left(n-n_{l, i}\right) S\left(n-n_{l, i}\right) \\
& \times C_{\hat{k}}\left(n-n_{\hat{l}}\right) S^{*}\left(n-n_{\hat{l}}\right) .
\end{aligned}
$$

$U_{\mathrm{AWGN}, \hat{l}, \hat{k}}(m)$ is noise component

$U_{\mathrm{AWGN}, \hat{l}, \hat{k}}(m)=\sum_{n=m N+n_{\hat{\imath}}}^{(m+1) N+n_{\hat{\imath}}-1} \eta_{\hat{l}}(n) C_{\hat{k}}\left(n-n_{\hat{\imath}}\right) S^{*}\left(n-n_{\hat{l}}\right)$.

In the $s$ th stage $(s>1)$, the multipath interference and the pilot interference terms are regenerated with the estimated fading gains and phases obtained from the previous stage $\left\{\hat{z}_{\hat{l}, \text { Pilot }}^{(s-1)}\right\}$. The regenerated interference terms are

$$
\begin{aligned}
\hat{U}_{\text {Pilot }, \hat{l}, \hat{k}}^{(s)}(m)= & \frac{\sqrt{\beta E_{D}}}{2} \sum_{\substack{l=1 \\
l \neq \hat{l}}}^{L_{R}} \hat{z}_{l, \text { Pilot }}^{(s-1)} \\
& \times \sum_{i=-\infty}^{\infty} \sum_{n=m N+n_{\hat{l}}}^{(m+1) N+n_{\hat{\imath}}-1} R\left(\bar{\tau}_{l, \hat{l}}-i T_{c}\right) \\
& \times D_{\text {Pilot }} C_{\text {Pilot }}\left(n-n_{l, i}\right) S\left(n-n_{l, i}\right) \\
& \times C_{\hat{k}}\left(n-n_{\hat{l}}\right) S^{*}\left(n-n_{\hat{l}}\right)
\end{aligned}
$$

and

$$
\begin{aligned}
\hat{U}_{p, \hat{l}, \hat{k}}^{(s)}(m)= & \frac{\sqrt{E_{D}}}{2} \sum_{k=1}^{K} \sum_{\substack{l=1 \\
l \neq \hat{l}}}^{L_{R}} \hat{z}_{l, \text { Pilot }}^{(s-1)} \\
& \times \sum_{i=-\infty}^{\infty} \sum_{n=m N+n_{\hat{\imath}}}^{(m+1) N+n_{\hat{\imath}}-1} R\left(\bar{\tau}_{l, \hat{l}}-i T_{c}\right) \\
& \times \hat{D}_{k}^{(s-1)}\left(m_{l, i}\right) C_{k}\left(n-n_{l, i}\right) S\left(n-n_{l, i}\right) \\
& \times C_{\hat{k}}\left(n-n_{\hat{l}}\right) S^{*}\left(n-n_{\hat{\imath}}\right)
\end{aligned}
$$

where $\hat{D}_{k}^{(s-1)}$ is the data decision from the $(s-1)$ th stage for the $k$ th code channel.

Then, the output of the interference cancellation unit of the sth stage is

$$
\begin{aligned}
z_{\hat{l}, \hat{k}}^{(s)}= & U_{s, \hat{l}, \hat{k}}(m)+U_{p, \hat{l}, \hat{k}}^{(1)}(m)-\hat{U}_{p, \hat{l}, \hat{k}}^{(s)}(m) \\
& +U_{\text {Pilot }, \hat{l}, \hat{k}}^{(1)}(m)-\hat{U}_{\mathrm{Pilot}, \hat{l}, \hat{k}}^{(s)}(m)+U_{\mathrm{AWGN}, \hat{l}, \hat{k}}(m) \\
= & U_{s, \hat{l}, \hat{k}}(m)+U_{p, \hat{l}, \hat{k}}^{(s)}(m)+U_{\mathrm{Pilot}, \hat{l}, \hat{k}}^{(s)}(m) \\
& +U_{\mathrm{AWGN}, \hat{l}, \hat{k}}(m) .
\end{aligned}
$$

\section{Pilot Channel Aided Coherent Demodulation}

In WCDMA standard [1], the pilot and data channels use different values of spreading factor (SF). The SF for the pilot channel $N_{\text {Pilot }}$ is fixed to 256 , whereas SF for the data channel $N$ is flexible from four to 256 . Similar to that of data channels, the output of the pilot channel for the $\hat{l}$ th branch in the $s$ th stage is written as

$$
\begin{aligned}
z_{\hat{\imath}, \mathrm{Pilot}}^{(s)}\left(n_{p}\right)= & N_{\text {Pilot }} \alpha_{\hat{l}} \sqrt{\beta E_{D}} D_{\text {pilot }} e^{j \xi_{\hat{\imath}}}+U_{p, \hat{l}, \text { Pilot }}^{(s)}\left(n_{p}\right) \\
& +U_{\text {Pilot, }, \hat{l}, \text { Pilot }}^{(s)}\left(n_{p}\right)+U_{\text {AWGN, }, \hat{l}, \text { Pilot }}\left(n_{p}\right)
\end{aligned}
$$

where $U_{\mathrm{AWGN}, \hat{l}, \text { Pilot }}\left(n_{p}\right)$ is the noise component, given by

$$
\begin{aligned}
U_{\mathrm{AWGN}, \hat{l}, \text { Pilot }}\left(n_{p}\right)=\sum_{n=n_{p} N_{\mathrm{Pilot}}+n_{\hat{\imath}}}^{\left(n_{p}+1\right) N_{\mathrm{Pilot}}+n_{\hat{\imath}}-1} \eta_{\hat{l}}(n) C_{\text {Pilot }}\left(n-n_{\hat{l}}\right) \\
\times S^{*}\left(n-n_{\hat{l}}\right) .
\end{aligned}
$$

$U_{p, \hat{l}, \text { Pilot }}^{(s)}\left(n_{p}\right)$ and $U_{\text {Pilot, }, \hat{j}, \text { Pilot }}^{(s)}\left(n_{p}\right)$ are residual multipath interference terms from other code channels and pilot channel, respectively. For the initial stage (first stage), these two types of interference cannot be canceled, which can be written as

$$
\begin{aligned}
& U_{p, \hat{l}, \text { Pilot }}^{(1)}\left(n_{p}\right)=\frac{\sqrt{E_{D}}}{2} \sum_{\substack{k=1 \\
l}}^{L} \sum_{\substack{l=1 \\
l \neq \hat{l}}}^{L} \alpha_{l} e^{j \xi_{l}} \\
& \times \sum_{i=-\infty}^{\infty} \sum_{n=n_{p} N_{\text {Pilot }}+n_{\hat{\imath}}}^{\left(n_{p}+1\right)} R\left(\bar{\tau}_{l, \hat{l}}-i T_{c}\right) \\
& \times D_{k}\left(m_{l, i}\right) C_{k}\left(n-n_{l, i}\right) S\left(n-n_{l, i}\right) \\
& \times C_{\text {Pilot }}\left(n-n_{\hat{l}}\right) S^{*}\left(n-n_{\hat{l}}\right)
\end{aligned}
$$

and

$$
\begin{aligned}
U_{\text {Pilot, }, \text {,Pilot }}^{(1)}\left(n_{p}\right)= & \frac{\sqrt{\beta E_{D}}}{2} \sum_{\substack{l=1 \\
l \neq \hat{l}}}^{L} \alpha_{l} e^{j \xi_{l}} \\
& \times \sum_{i=-\infty}^{\infty} \sum_{n=n_{p} N_{\text {Pilot }}+n_{\hat{\imath}}}^{\left(n_{p}+1\right) N_{\text {Pilot }}+n_{\hat{\imath}}-1} R\left(\bar{\tau}_{l, \hat{l}}-i T_{c}\right) \\
& \times D_{\text {Pilot }} C_{\text {Pilot }}\left(n-n_{l, i}\right) S\left(n-n_{l, i}\right) \\
& \times C_{\text {Pilot }}\left(n-n_{\hat{l}}\right) S^{*}\left(n-n_{\hat{l}}\right) .
\end{aligned}
$$


In the $s$ th stage, the residual interference can be regenerated by the demodulated data and channel estimate from the previous stage, i.e.,

$$
\begin{aligned}
& U_{p, \hat{l}, \text { Pilot }}^{(s)}\left(n_{p}\right) \\
& =\frac{\sqrt{E_{D}}}{2} \\
& \times \sum_{k=1}^{K} \sum_{\substack{l=1 \\
l \neq \hat{l}}}^{L} \alpha_{l} e^{j \xi_{l}} \sum_{i=-\infty}^{\infty} \sum_{n=n_{p} N_{\text {Pilot }}+n_{\hat{l}}}^{\left(n_{p}+1\right) N_{\text {Pilot }}+n_{\hat{l}}-1} R\left(\bar{\tau}_{l, \hat{l}}-i T_{c}\right) \\
& \times D_{k}\left(m_{l, i}\right) C_{k}\left(n-n_{l, i}\right) S\left(n-n_{l, i}\right) \\
& \times C_{\text {Pilot }}\left(n-n_{\hat{l}}\right) S^{*}\left(n-n_{\hat{l}}\right) \\
& -\frac{\sqrt{E_{D}}}{2} \\
& \times \sum_{k=1}^{K} \sum_{\substack{l=1 \\
l \neq \hat{l}}}^{L_{R}} \hat{z}_{l, \text { Pilot }}^{(s-1)} \sum_{i=-\infty}^{\infty} \sum_{n=n_{p} N_{\text {Pilot }}+n_{\hat{l}}}^{\infty} R\left(\bar{\tau}_{l, \hat{l}}-i T_{c}\right) \\
& \times \hat{D}_{k}^{(s-1)}\left(m_{l, i}\right) C_{k}\left(n-n_{l, i}\right) S\left(n-n_{l, i}\right) \\
& \text { and } \times C_{\text {Pilot }}\left(n-n_{\hat{l}}\right) S^{*}\left(n-n_{\hat{l}}\right) \\
& U_{\text {Pilot, }, \text {,Pilot }}^{(s)}\left(n_{p}\right) \\
& =\frac{\sqrt{\beta E_{D}}}{2} \sum_{\substack{l=1 \\
l \neq \hat{l}}}^{L} \alpha_{l} e^{j \xi_{l}} \sum_{i=-\infty}^{\infty} \sum_{n=n_{p} N_{\text {Pilot }}+n_{\hat{l}}}^{\left(n_{p}+1\right) N_{\text {Pilot }}+n_{\hat{\imath}}-1} R\left(\bar{\tau}_{l, \hat{l}}-i T_{c}\right) \\
& \times D_{\text {Pilot }} C_{\text {Pilot }}\left(n-n_{l, i}\right) S\left(n-n_{l, i}\right) \\
& \times C_{\text {Pilot }}\left(n-n_{\hat{l}}\right) S^{*}\left(n-n_{\hat{l}}\right) \\
& -\frac{\sqrt{\beta E_{D}}}{2} \\
& \times \sum_{\substack{l=1 \\
l \neq \hat{l}}}^{L_{R}} \hat{z}_{l, \text { Pilot }}^{(s-1)} \sum_{i=-\infty}^{\infty} \sum_{n=n_{p} N_{\text {Pilot }}+n_{\hat{l}}}^{\infty} R\left(\bar{\tau}_{l, \hat{l}}-i T_{c}\right) \\
& \times D_{\text {Pilot }} C_{\text {Pilot }}\left(n-n_{l, i}\right) S\left(n-n_{l, i}\right) \\
& \times C_{\text {Pilot }}\left(n-n_{\hat{l}}\right) S^{*}\left(n-n_{\hat{l}}\right) \text {. }
\end{aligned}
$$

Then, the scaled fading estimation is $\hat{z}_{\hat{l}, \mathrm{Pilot}}^{(s)}\left(n_{p}\right)=$ $z_{\hat{l}, \text { Pilot }}^{(s)}\left(n_{p}\right) / N_{\text {Pilot }} \sqrt{\beta E_{D}} D_{\text {Pilot }}$, which is used for symbol detection in the $s$ th stage. In order to improve the channel estimation, a simple $W$-tap FIR filter is used, i.e.,

$$
\hat{z}_{\hat{l}, \text { Pilot }}^{(s)}(m)=\sum_{\text {tap }=-\lfloor(W-1) / 2\rfloor+1}^{\lfloor W / 2\rfloor} \frac{z_{\hat{l}, \text { Pilot }}^{(s)}(m-t a p)}{W N_{\text {Pilot }} \sqrt{\beta E_{D}} D_{\text {Pilot }}} .
$$

Therefore, the scaled outputs of the coherent Rake receiver for the $\hat{k}$ th code channel in the $s$ th stage are as shown in (24) at the bottom of the page and in (25) at the bottom of the page, where $\Re\{x\}$ and $\Im\{x\}$ stand for real and imaginary parts, respectively.

The decision block will detect the in-phase and quadrature phase of the transmitted QAM symbol from (25) and (26) with the boundary corresponding to M-QAM constellation as shown in [14]. Without loss of generality, we will analyze the BER performance for the first code channel in the following.

\section{BER PeRformance ANALYsis}

As the outputs of the in-phase and quadrature phase channels are symmetric, we will focus only on the in-phase channel output at the receiver. Concerning the decision variable $\Re\left\{Y_{1}^{(s)}(m)\right\}$, we derive the average BER over all the possible M-QAM constellation points in the first quadrant. At first, we start with the 16-QAM modulation, and a general analytical BER performance for M-QAM will be presented afterwards.

Similar to [14], for 16-QAM modulated WCDMA systems, when the transmitted data for the in-phase channel are $\Re\left\{D_{1}(m)\right\}=3 / \sqrt{5 / 2}$, i.e., the most significant bit (MSB) of the transmitted bit-pair in the in-phase channel is one and the least significant bit (LSB) is also one, it is found that an error occurs only on the MSB if $\Re\left\{Y_{1}^{(s)}(m)\right\}<-2 / \sqrt{5 / 2}$ and only on the LSB if $0 \leq \Re\left\{Y_{1}^{(s)}(m)\right\}<2 / \sqrt{5 / 2}$. However, when $-2 / \sqrt{5 / 2} \leq \Re\left\{Y_{1}^{(s)}(m)\right\}<0$, both the LSB and the

$$
\Re\left\{Y_{\hat{k}}^{(s)}(m)\right\}=\frac{\frac{1}{2} \sum_{l=1}^{L_{R}}\left(z_{l, \hat{k}}^{(s)}(m)\left(\hat{z}_{l, \mathrm{Pilot}}^{(s)}(m)\right)^{*}+\left(z_{l, \hat{k}}^{(s)}(m)\right)^{*} \hat{z}_{l, \mathrm{Pilot}}^{(s)}(m)\right)}{N \sqrt{E_{D}} \sum_{l=1}^{L_{R}}\left|\hat{z}_{l, \mathrm{Pilot}}^{(s)}(m)\right|^{2}}
$$

$$
\Im\left\{Y_{\hat{k}}^{(s)}(m)\right\}=\frac{\frac{-j}{2} \sum_{l=1}^{L_{R}}\left(z_{l, \hat{k}}^{(s)}(m)\left(\hat{z}_{l, \mathrm{Pilot}}^{(s)}(m)\right)^{*}-\left(z_{l, \hat{k}}^{(s)}(m)\right)^{*} \hat{z}_{l, \mathrm{Pilot}}^{(s)}(m)\right)}{N \sqrt{E_{D}} \sum_{l=1}^{L_{R}}\left|\hat{z}_{l, \mathrm{Pilot}}^{(s)}(m)\right|^{2}}
$$


MSB are demodulated incorrectly, i.e., the conditional BER is given by

$$
\begin{aligned}
& p^{(s)}\left(e \mid D_{1}^{I}=\frac{3}{\sqrt{\frac{5}{2}}}, D_{1}^{Q}\right) \\
& =\frac{1}{2}\left(p\left|0 \leq \Re\left\{Y_{1}^{(s)}\right\}<\frac{2}{\sqrt{\frac{5}{2}}}\right| D_{1}^{I}=\frac{3}{\sqrt{\frac{5}{2}}}, D_{1}^{Q}\right) \\
& +2^{*} p\left(-\frac{2}{\sqrt{\frac{5}{2}}} \leq \Re\left\{Y_{1}^{(s)}\right\}<0 \mid D_{1}^{I}=\frac{3}{\sqrt{\frac{5}{2}}}, D_{1}^{Q}\right) \\
& \left.+p\left(\Re\left\{Y_{1}^{(s)}\right\}<-\frac{2}{\sqrt{\frac{5}{2}}} \mid D_{1}^{I}=\frac{3}{\sqrt{\frac{5}{2}}}, D_{1}^{Q}\right)\right) \\
& =\frac{1}{2}\left(p\left(\Re\left\{Y_{1}^{(s)}\right\}<\frac{2}{\sqrt{\frac{5}{2}}} \mid D_{1}^{I}=\frac{3}{\sqrt{\frac{5}{2}}}, D_{1}^{Q}\right)\right. \\
& +p\left(\Re\left\{Y_{1}^{(s)}\right\}<0 \mid D_{1}^{I}=\frac{3}{\sqrt{\frac{5}{2}}}, D_{1}^{Q}\right) \\
& \left.-p\left(\Re\left\{Y_{1}^{(s)}\right\}<-\frac{2}{\sqrt{\frac{5}{2}}} \mid D_{1}^{I}=\frac{3}{\sqrt{\frac{5}{2}}}, D_{1}^{Q}\right)\right)
\end{aligned}
$$

where $D_{1}^{I}$ and $D_{1}^{Q}$ are real and imaginary parts of $D_{1}$, respectively; " $m$ " is simply omitted, unless otherwise noted. Similarly, we can get the conditional BER when $D_{1}^{l}=1 / \sqrt{5 / 2}$

$$
\begin{aligned}
p^{(s)}\left(e \mid D_{1}^{I}=\frac{1}{\sqrt{\frac{5}{2}}}, D_{1}^{Q}\right) \\
=\frac{1}{2}\left(1-p\left(\Re\left\{Y_{1}^{(s)}\right\}<\frac{2}{\sqrt{\frac{5}{2}}} \mid D_{1}^{I}=\frac{1}{\sqrt{\frac{5}{2}}}, D_{1}^{Q}\right)\right. \\
+p\left(\Re\left\{Y_{1}^{(s)}\right\}<0 \mid D_{1}^{I}=\frac{1}{\sqrt{\frac{5}{2}}}, D_{1}^{Q}\right) \\
\left.+p\left(\Re\left\{Y_{1}^{(s)}\right\}<-\frac{2}{\sqrt{\frac{5}{2}}} \mid D_{1}^{I}=\frac{1}{\sqrt{\frac{5}{2}}}, D_{1}^{Q}\right)\right)
\end{aligned}
$$

Therefore, the probability of error of the $s$ th stage $p_{b}^{(s)}$ is given by

$$
p_{b}^{(s)}=\frac{1}{4} \sum_{p=1}^{2} \sum_{q=1}^{2} p^{(s)}\left(e \mid D_{1}^{I}=\frac{2 p-1}{\sqrt{\frac{5}{2}}}, D_{1}^{Q}=\frac{2 q-1}{\sqrt{\frac{5}{2}}}\right) .
$$

The above probability can be evaluated by calculating the common probability, i.e., $p\left(\hat{D}_{1}^{I(s)}<\tilde{B} \mid D_{1}\right)$, $\tilde{B}=\{2,0,-2\} / \sqrt{5 / 2}$ for 16-QAM case. It is a special case of the general quadratic form in $[19$, Appendix $\mathrm{B}]$, where $A=0$, $B=-\tilde{B} N \sqrt{E_{D}}$, and $C=1 / 2$.
Therefore, by averaging the conditional probabilities over all the possible constellation points in the first quadrant, we can get a general formula to calculate the BER performance of M-QAM, where each symbol represents $\log _{2} M$ bits information

$$
\begin{aligned}
& p_{b}^{(s)} \\
& =\frac{1}{\frac{M}{4}} \sum_{p=1}^{\sqrt{M / 2}} \sum_{q=1}^{\sqrt{M / 2}} p^{(s)} \\
& \quad \times\left(e \mid D_{1}^{I}=\frac{2 p-1}{\left.\sqrt{\frac{2(M-1)}{\left(3 \log _{2} M\right)}}, D_{1}^{Q}=\frac{2 q-1}{\sqrt{\frac{2(M-1)}{\left(3 \log _{2} M\right)}}}\right)}\right.
\end{aligned}
$$

and the conditional probability $p^{(s)}\left(e \mid D^{I}, D^{Q}\right)$ can be calculated in a similar way as that for 16-QAM.

To obtain the BER performance, we need to calculate the conditional probability $p\left(\Re\left\{Y_{1}^{(s)}\right\}<\tilde{B} \mid D_{1}\right)$. By directly applying the characteristic function method, we obtain the conditional probability as

$$
p\left(\Re\left\{Y_{1}^{(s)}\right\}<\tilde{B} \mid D_{1}\right)=-\frac{1}{2 \pi j} \int_{-\infty+j \sigma}^{\infty+j \sigma} \frac{\phi(j v)}{v} d v
$$

where $\sigma$ is a small positive number and $\phi(j v)$ is the characteristic function of the decision variable $\left(\left(\Re\left\{Y_{1}^{(s)}\right\}-\tilde{B}\right) N \sqrt{E_{D}} \sum_{l=1}^{L_{R}}\left|\hat{z}_{l, \text { Pilot }}^{(s)}\right|^{2}\right), \quad$ conditioned on a given transmitted symbol $D_{1}$. Equation (30) is valid, no matter whether the fading channels have equal or unequal fading power. Referring to [19, Appendix B], we must get the first and second moment statistic functions of $z_{i, 1}^{(s)}$ and $\hat{z}_{i \text {,Pilot }}^{(s)}$ in order to derive the conditional BER.

In Rayleigh fading channel environments, the mean of $z_{i, 1}^{(s)}$ and $\hat{z}_{i \text {,Pilot }}^{(s)}$ are zero. The characteristic function is rewritten as

$$
\phi(j v)=\prod_{l=1}^{L_{R}} \frac{v_{1 l} v_{2 l}}{\left(v+j v_{1 l}\right)\left(v-j v_{2 l}\right)}
$$

where

$$
\begin{aligned}
v_{1 l}, v_{2 l} & =\mp v_{3 l}+\sqrt{\frac{v_{3 l}^{2}+1}{\left(R_{z_{l} z_{l} \mid D_{1}}^{(s)} R_{\hat{z}_{l} \hat{z}_{l} \mid D_{1}}^{(s)}-\left|R_{z_{l} \hat{z}_{l} \mid D_{1}}^{(s)}\right|^{2}\right)}} \\
v_{3 l} & =\frac{\left(-\tilde{B} N \sqrt{E_{D}} R_{\hat{z}_{l} \hat{z}_{l} \mid D_{1}}^{(s)}+\Re\left\{R_{z_{l} \hat{z}_{l} \mid D_{1}}^{(s)}\right\}\right)}{\left(R_{z_{l} z_{l} \mid D_{1}}^{(s)} R_{\hat{z}_{l} \hat{z}_{l} \mid D_{1}}^{(s)}-\left|R_{z_{l} \hat{z}_{l} \mid D_{1}}^{(s)}\right|^{2}\right)}
\end{aligned}
$$

$R_{z_{l} z_{l} \mid D_{1}}^{(s)}, R_{\hat{z}_{l} \hat{z}_{l} \mid D_{1}}^{(s)}$, and $R_{z_{l} \hat{z}_{l} \mid D_{1}}^{(s)}$ are second moment statistic functions of $z_{l, 1}^{(s)}$ and $\hat{z}_{l, \text { Pilot }}^{(s)}$.

Now, we have to obtain the second statistical functions $R_{z_{i} z_{i} \mid D_{1}(m)}^{(s)}, R_{\hat{z}_{i} \hat{z}_{i} \mid D_{1}(m)}^{(s)}$, and $R_{z_{i} \hat{z}_{i} \mid D_{1}(m)}^{(s)}$. Assuming that the combination of each channelization code with scrambling code is a pure random sequence, thanks to the long scrambling 
code deployed in 3GPP, so as to the time shift of the combined sequence. Therefore, the number of independent interference components from each propagation path is $K+1$, and the total number of independent interference components in the despreading output for each data channel or the pilot channel is $(K+1)(L-1)$. Here, the interference from other code channels in the same path is zero due to the orthogonality of the channel codes. Thus, according to central limit theorem, when $(K+1)(L-1) \gg 1$, the overall multipath interference from all code channels can be Gaussian approximated. Similar to the derivation for the variance of the multipath interference [8], one obtains

$$
\begin{aligned}
R_{z_{l} z_{l} \mid D_{1}}^{(1)}= & \frac{E\left\{z_{l, 1}^{(1)}\left(z_{l, 1}^{(1)}\right)^{*} \mid D_{1}\right\}}{2} \\
= & \frac{N^{2} E_{D}\left|D_{1}\right|^{2} \Omega_{l}}{2} \\
& +\frac{N E_{D}\left(K \log _{2} M+2 \beta\right)}{3} \\
& \times \sum_{\substack{l 1=1 \\
l 1 \neq l}}^{L} \Omega_{l 1}+N N_{0} \\
R_{\hat{z}_{l} \hat{z}_{l} \mid D_{1}}^{(1)}= & \frac{E\left\{\hat{z}_{l, 1}^{(1)}\left(\hat{z}_{l, 1}^{(1)}\right)^{*} \mid D_{1}\right\}}{2} \\
= & \frac{\Omega_{l}}{2}+\frac{\left(K \log _{2} M+2 \beta\right)}{\left(6 W N_{\text {Pilot }} \beta\right)} \\
& \times \sum_{\substack{l 1=1 \\
l 1 \neq l}}^{L} \Omega_{l 1}+\frac{N_{0}}{\left(2 W N_{\text {Pilot }} \beta E_{D}\right)}
\end{aligned}
$$

and

$$
R_{z_{l} \hat{z}_{l} \mid D_{1}}^{(1)}=\frac{E\left\{z_{l, 1}^{(1)}\left(\hat{z}_{l, 1}^{(1)}\right)^{*} \mid D_{1}\right\}}{2}=\frac{N \sqrt{E_{D}} D_{1} \Omega_{l}}{2} .
$$

In the following stages, the second moment statistic function $R_{z_{l} \hat{z}_{l} \mid D_{1}(m)}^{(s)}$ is the same as (36). As for the second moment statistical function of $z_{i, 1}^{(s)}$, the interference from the $L-L_{R}$ paths is not cancelled-only the interference from the $L_{R}$ strongest paths is being cancelled. However, due to the imperfect channel estimation and the incorrect tentative symbol detection in the previous stage, the regenerated interference is not exactly the interference. Thus, the residual interference occurs due to imperfect channel estimation and incorrect symbol detection, as a portion of the product of symbol error and channel estimation error. It is difficult to analyze their effects jointly. Fortunately, because the channel estimation error results from the effects of imperfect channel estimation and symbol detection in the previous stage over the recent $W N_{\text {Pilot }}$ chips, it has weak correlation with the symbol detection error for only one time instance $m$, i.e., over $N$ chips, where $N \ll W N_{\text {Pilot. }}$. So the channel estimation and the tentative data decisions are approximated as uncorrelated to each other [8], we obtain

$$
\begin{aligned}
R_{z_{l} z_{l} \mid D_{1}}^{(s)}= & \frac{N^{2} E_{D}\left|D_{1}\right|^{2} \Omega_{l}}{2}+\frac{N E_{D}}{3} \\
& \times \sum_{k=1}^{K} \sum_{\substack{l 1=1 \\
l 1 \neq l}}^{L_{R}}\left(E\left\{\left|D_{k}-\hat{D}_{k}^{(s-1)}\right|^{2}\right\} \Omega_{l 1}\right. \\
& \left.+8 \log _{2} M\left(\sigma_{e, l 1}^{(s-1)}\right)^{2}\right) \\
& +\frac{16 N \beta E_{D}}{3} \sum_{\substack{l 1=1 \\
l 1 \neq l}}^{L_{R}}\left(\sigma_{e, l 1}^{(s-1)}\right)^{2} \\
& +\frac{N E_{D}\left(K \log _{2} M+2 \beta\right)}{6} \sum_{l 1=L_{R}+1}^{L} \Omega_{l 1}+N N_{0}
\end{aligned}
$$

where the last two components on the right-hand side of the first line are the variances of the residual interference of the $L_{R}$ strongest paths from data channel resulting from the imperfect tentative decisions with channel estimation error-free from the previous stage and the imperfect channel estimates with symbol decision error-free, respectively.

The variance of tentative data decision is shown in (38) at the bottom of the page and the variance of channel estimation error in the previous stage $\left(\sigma_{e, l}^{s-1}\right)^{2}$ is $R_{\hat{z}_{l}, \hat{z}_{l} \mid D_{1}}^{s-1}-\Omega_{l} / 2$. As to the second statistical function of the estimated fading, it is given by

$$
\begin{aligned}
R_{\hat{z}_{l} \hat{z}_{l} \mid D_{1}}^{(s)}= & \frac{\Omega_{l}}{2}+\frac{1}{\left(6 W N_{\text {Pilot }} \beta\right)} \\
& \times \sum_{k=1}^{K} \sum_{\substack{l 1=1 \\
l 1 \neq l}}^{L_{R}}\left(E\left\{\left|D_{k}-\hat{D}_{k}^{(s-1)}\right|^{2}\right\} \Omega_{l 1}\right. \\
& \left.+8 \log _{2} M\left(\sigma_{e, l 1}^{(s-1)}\right)^{2}\right) \\
& \frac{8}{\left(3 W N_{\text {Pilot }}\right)} \sum_{\substack{l 1=1 \\
l 1 \neq l}}^{L_{R}}\left(\sigma_{e, l 1}^{(s-1)}\right)^{2} \\
& +\frac{\left(K \log _{2} M+2 \beta\right)}{\left(6 W N_{\text {Pilot }} \beta\right)} \\
& \times \sum_{l 1=L_{R}+1}^{L} \Omega_{l 1}+\frac{N_{0}}{\left(2 W N_{\text {Pilot }} \beta E_{D}\right)} .
\end{aligned}
$$

After calculating (30) by using Gauss-Chebyshev quadrature method [21], the BER (29) is achieved.

$$
E\left\{\left|D_{k}-\hat{D}_{k}^{(s-1)}\right|^{2}\right\}=\sum_{D_{k}} \sum_{\hat{D}_{k}^{(s-1)}} \frac{\left|D_{k}-\hat{D}_{k}^{(s-1)}\right|^{2} p\left(\hat{D}_{k}^{I(s-1)} \mid D_{k}\right) p\left(\hat{D}_{k}^{Q(s-1)} \mid D_{k}\right)}{M^{2}}
$$




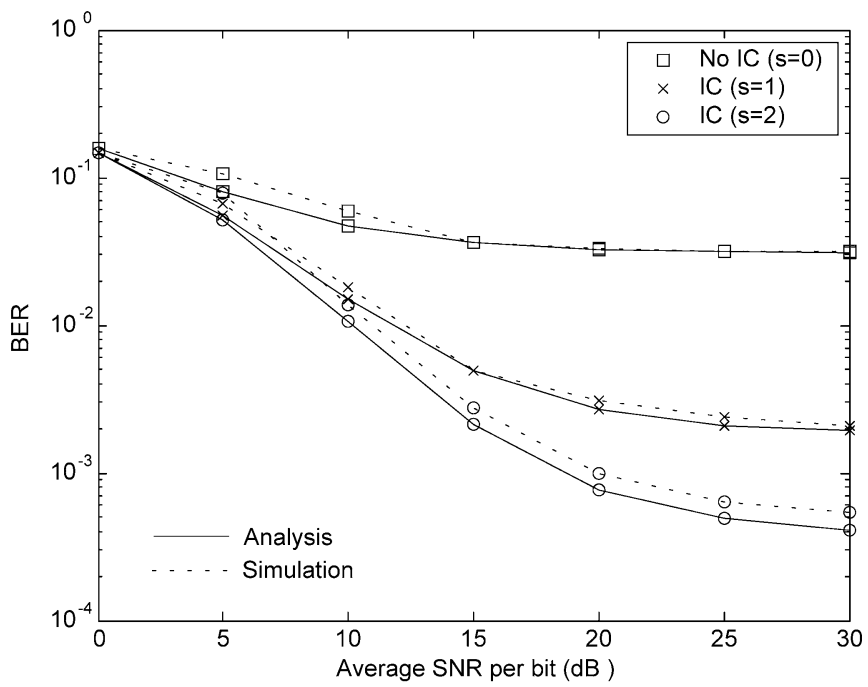

(a) 4-QAM

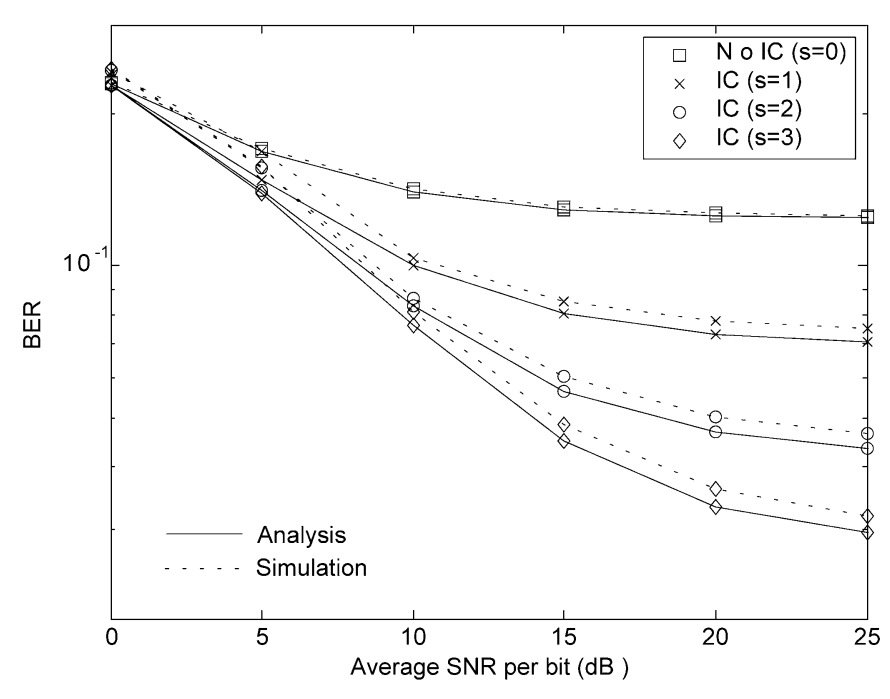

(b) 16-QAM

Fig. 4. BER versus average received SNR per bit for M-QAM multicode CDMA systems.

\section{NuMERICAL RESULTS}

In this section, some representative numerical results are presented which illustrate potential of using the multistage parallel interference cancellation as an effective tool for improving the performance of M-QAM modulated multicode WCDMA systems over multipath fading channels. The effect of different system parameters such as noise and the power ratio of the pilot channel to a data channel on the system are investigated by numerical evaluation. A six-path channel (indoor channel B model in [22]) is used. Unless noted otherwise, the spread factors for data channels and the pilot channel are 16 and 256, respectively, the number of code channels $K$ is six, the number of Rake fingers $L_{R}$ is three, the length of the FIR filter for channel estimation $W$ is three, and the power ratio of pilot to one data channel $\beta$ is $2 \mathrm{~dB}$.

Fig. 4 shows the BER performance versus signal-to-noise ratio (SNR) per bit for different stages of interference cancellation and different modulations with perfect channel estimation. From the figure, it can be seen that the BER performance in the initial stage (first stage) is seriously limited by the multipath interference from the code channels. The interference cancellation method dramatically improves the performance in the following stages. Comparing the performance curves of 4-QAM with those of 16-QAM, it can be seen that much more interference cancellation stages are required for the 16-QAM system. Because the minimal distance $\left(2 \sqrt{3 \log _{2} M /(2(M-1))}\right)$ between constellation points is smaller, the higher order QAM system is very sensitive to the additive multipath interference. It is found that in higher SNR cases, higher order modulation can be adaptively employed to increase the system throughput. Meanwhile, to illustrate the accuracy of the analytical method, some simulation results are plotted in Fig. 4. It can be observed that simulation results are close to analytical results in the first some stages. The gap between analytical curves and simulation curves becomes larger with the increase of interference cancellation stages. This is because of the inaccurate Gaussian approx- imation of the residual multipath interference when the number of stages is larger.

In WCDMA systems, the power of pilot channel is an important parameter for system performance. Basically, by increasing the pilot power, the effect of the multipath interference from data channels to the pilot channel will be decreased, which results in more accurate channel estimation. However, too much pilot power will cause more residual multipath interference to the data channels from pilot channel, which will also degrade the system performance. So a tradeoff must exist in the allocation of the pilot channel power. Fig. 5 shows the BER performance of systems without [Fig. 5(a)] and with [Fig. 5(b)] interference cancellation for 16-QAM when the average SNR per bit is 20 $\mathrm{dB}$. It can be seen that the performance improves when $\beta$ increases from $-10 \mathrm{~dB}$. After $\beta$ reaches its optimal value, the performance degrades with the increase of $\beta$ afterwards. Moreover, channel estimation not only affects the fading compensation but also determines the accuracy of regenerated interference in the following stages. It can be observed that without interference cancellation $(s=0)$, the optimum $\beta$ are about -1 and $0 \mathrm{~dB}$ for $K=4$ and 6 , respectively, and when $s=1$, the optimum $\beta$ are about 3 and $4 \mathrm{~dB}$ for $K=4$ and 6 , respectively. It implies that for 16-QAM, the power ratio of pilot channel to the total transmitted power $(\beta / \beta+2 K)$ is about $8 \%$ and $18 \%$ for $s=1$ and 1 , respectively. So to achieve more accurate channel estimation, the power ratio for systems with interference cancellation should be much larger than that for systems without interference cancellation.

\section{CONCLUSION}

In this paper, we have studied the performance of QAM modulated multicode CDMA systems with interference cancellation for high data rate service. The analytical BER of the receiver considering the imperfect channel estimation over multipath Rayleigh fading channels is derived. Simulation results are presented to illustrate the accuracy of the analytical results. Numerical results also show that interference cancellation is an 


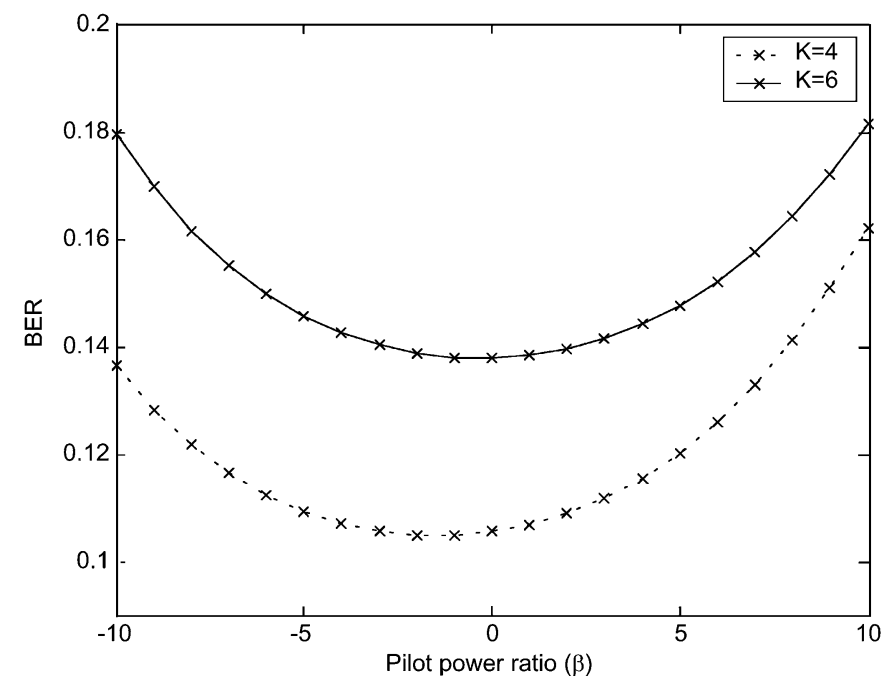

(a) Without interference cancellation $(s=0)$

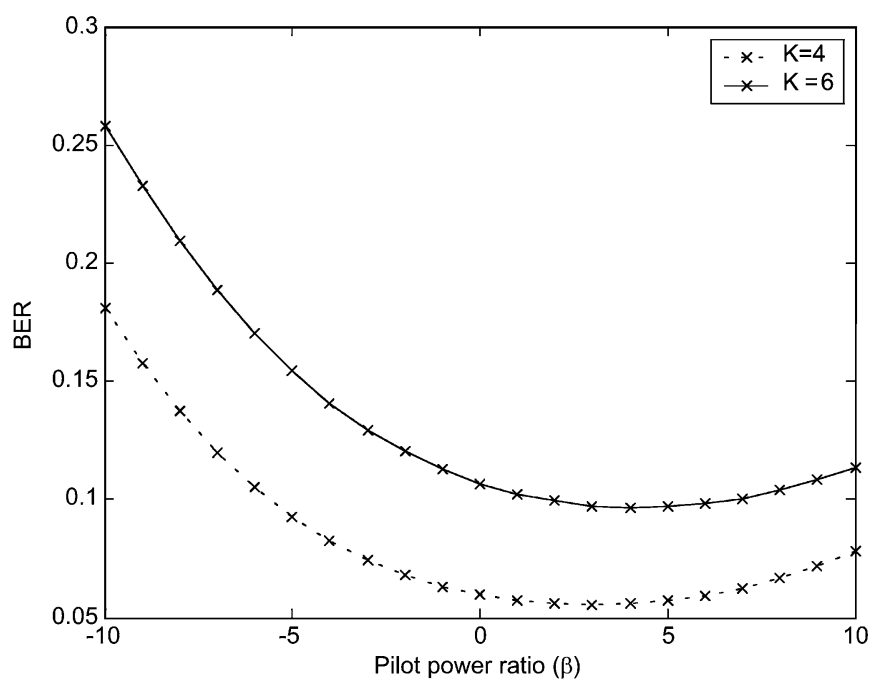

(b) With interference cancellation $(s=1)$

Fig. 5. Effect of pilot channel power on BER performance for 16-QAM multicode CDMA systems.

efficient technique for M-QAM multicode CDMA system. With interference cancellation, for 16-QAM systems, optimal value for power ratio of the pilot channel to data channels is a bit larger than that for the system without interference cancellation. It implies that extra pilot power should be invested for more accurate channel estimation, so that better BER performance can be achieved when using the interference cancellation technique.

\section{ACKNOWLEDGMENT}

The authors would like to thank Dr. M. Sawahashi and Dr. K. Higuchi of NTT DoCoMo, Japan, for their helpful discussion of QAM to multicode CDMA.

\section{REFERENCES}

[1] "Spreading and modulation (FDD),", Tech. Spec. 3GPP TS25.213 version 3.4.0, 3GPP TSG-RAN, 2000-12.

[2] F. Adachi, M. Sawahashi, and H. Suda, "Wideband DS-CDMA for nextgeneration mobile communications systems," IEEE Commun. Mag., vol. 36, pp. 56-69, Sep. 1998.

[3] M. K. Varanas and B. Aazhang, "Multistage detection in asynchronous code-division multiple-access communications," IEEE Trans. Commun., vol. 38, pp. 509-519, Apr. 1990.

[4] Y. C. Yoon, R. Kohno, and H. Imai, "A spread-spectrum multiaccess system with cochannel interference cancellation for multipath fading channels," IEEE J. Sel. Areas Commun., vol. 11, pp. 1067-1075, Sep. 1993.

[5] M. Mostofa, K. Howlader, and B. D. Woerner, "Iterative interference cancellation and decoding using a soft cancellation factor for DS-CDMA," in Proc. IEEE Veh. Tech. Conf., 2000, pp. 2076-2080.

[6] N. S. Correal, R. M. Buehrer, and B. D. Woerner, "Improved CDMA performance through bias reduction for parallel interference cancellation," in Proc. IEEE PIMRC'97, 1997, pp. 565-569.

[7] M. Sawahashi, H. Andoh, and K. Higuchi, "Interference rejection weight control for pilot symbol-assisted coherent multistage interference canceller using recursive channel estimation in DS-CDMA mobile radio," IEICE Trans. Fundamentals, vol. E81-A, pp. 957-972, May 1998.

[8] J. C. Chen, J. Wang, and M. Sawahashi, "MCI cancellation for multicode wideband CDMA systems," IEEE J. Sel. Areas Commun., vol. 20, pp. 450-462, Feb. 2002.
[9] L. B. Milstein and M. S. Lim, "On the performance of a higher order alphabet size in CDMA," IEEE Commun. Lett., vol. 1, pp. 9-11, Jan. 1997.

[10] P. Shamain and L. B. Milstein, "Minimum mean square error (MMSE) receiver employing 16-QAM in CDMA channel with narrowband Gaussian interference," in Proc. IEEE Military Commun. Conf. (MILCOM), 1999, pp. 826-830.

[11] J.-H. Perrin, S. Buljore, J. Zeidler, and L. B. Milstein, "Performance evaluation of space-path diversity and higher alphabet size for CDMA," in Proc. IEEE Signals, Syst. Computers Conf., 1997, pp. 197-201.

[12] S. Sampei and T. Sunaga, "Rayleigh fading compensation for QAM in land mobile radio communications," IEEE Trans. Veh. Technol., vol. 42, pp. 137-147, May 1993.

[13] T. Sunaga and S. Sampei, "Performance of multi-level QAM with postdetection maximal ratio combining space diversity for digital land-mobile radio communications," IEEE Trans. Veh. Technol., vol. 42, pp. 294-301, Aug. 1993.

[14] X. Tang, M.-S. Alouini, and A. J. Goldsmith, "Effect of channel estimation error on M-QAM BER performance in Rayleigh fading," IEEE Trans. Commun., vol. 47, pp. 1856-1864, Dec. 1999.

[15] A. Annamalai, C. Tellambura, and V. K. Bhargava, "Exact evaluation of maximal-ratio and equal-gain diversity receivers for M-ary QAM on Nakagami fading channels," IEEE Trans. Commun., vol. 47, pp. 1335-1344, Sep. 1999.

[16] D. Kim and V. K. Bhargava, "Performance of multidimensional multicode DS-CDMA using code diversity and error detection," IEEE Trans. Commun., vol. 49, pp. 875-887, May 2001.

[17] A. Annamalai and C. Tellambura, "Error rates for Nakagami-m fading multichannel reception of binary and M-ary signals," IEEE Trans. Commun., vol. 49, pp. 58-68, Jan. 2001.

[18] K. Higuchi, A. Fujiwara, and M. Sawahashi, "Multipath interference canceller for high-speed packet transmission with adaptive modulation and coding scheme in W-CDMA forward link," IEEE J. Sel. Areas Commun., vol. 20, pp. 419-432, Feb. 2002.

[19] J. G. Proakis, Digital Communications. New York: McGraw-Hill, 1995.

[20] J. Wang and J. Chen, "Performance of wideband CDMA systems with complex spreading and imperfect channel estimation," IEEE J. Sel. Areas Commun., vol. 19, pp. 152-163, Jan. 2001.

[21] J. R. Foerster and L. B. Milstein, "Coded modulation for a coherent DS-CDMA system employing an MMSE receiver in a fading channel," IEEE Trans. Commun., vol. 48, pp. 1909-1918, Nov. 2000.

[22] "Guidelines for evaluation of radio transmission technology for IMT2000,”, ITU-R M.1225, 1997. 


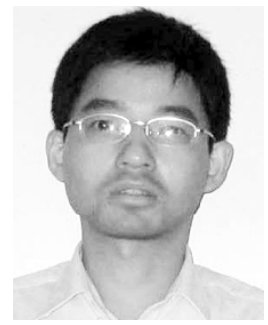

Bin Xia received the B.Eng. degree in electrical engineering and the M.Eng. degree in information and communication engineering from the University of Science and Technology of China, Hefei, in 1997 and 2000, respectively, and the Ph.D. degree in electrical engineering from the University of Hong Kong, China, in 2004.

From 1995 to 2000, he was with the Personal Communication and Spread Spectrum Laboratory, the University of Science and Technology of China, as a Research Engineer involved in the development of CDMA communication systems based on IS-95 and UMTS standards. From 1999 to 2001, he was with UTStarcom Inc. working on WCDMA systems. He is now a System Engineer with Alcatel Shanghai Bell Co. Ltd. His research interests are in the areas of broadband wireless access technologies, wireless networking, and VLSI implementation of wireless transceivers.

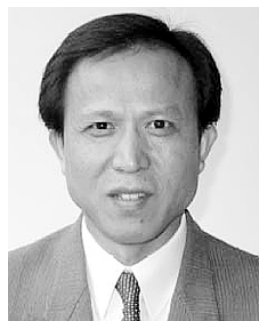

Jiangzhou Wang (M'91-SM'94) received the B.S. and M.S. degrees from Xidian University, Xian, China, in 1983 and 1985, respectively, and the Ph.D. degree (with greatest distinction) from the University of Ghent, Belgium, in 1990, all in electrical engineering.

From 1990 to 1992, he was a Postdoctoral Fellow with the University of California at San Diego, where he worked on the research and development of cellular CDMA systems. From 1992 to 1995, he was a Senior System Engineer with Rockwell International Corporation, Newport Beach, CA, where he worked on the development and system design of wireless communications. Since 1995, he has been with the University of Hong Kong, China, where he is currently an Associate Professor and the Coordinator of the Telecommunications Group. He has held a Visiting Professor position with NTT DoCoMo, Japan.

He was a Technical Chairman of IEEE Workshop in 3G Mobile Communications, 2000. He has published more than 100 papers, including more than 30 IEEE TRANSACTIONS/JOURNAL papers in the areas of wireless mobile and spread spectrum communications. He has written/edited two books: Broadband Wireless Communications (Norwell, MA: Kluwer, 2001) and Advances in $3 G$ Enhanced Technologies for Wireless Communications (Reading, MA: Artech House, 2002), respectively. He has received one U.S. patent in the GSM system.

Dr. Wang is an Editor for IEEE TRANSACTIONS ON COMMUNICATIONS and a Guest Editor for IEEE JOURNAL ON SELECTED AREAS IN COMMUNICATIONS (Wideband CDMA, August 2000 and January 2001; and Advances in Multicarrier CDMA, 2006). He is listed in Who's Who in the World. 